\title{
Thermal Analysis of Electromagnetic Engine Water Cooling System Based on Fluid-solid Coupling
}

\author{
Ruibin Zhang a, Guofu Wang, Yuanhua Chen \\ Guilin University of Aerospace Technology, Guilin 541004, China \\ a3504356595@qq.com
}

Keywords: Electromagnetic coils, Water cooling system, Fluid-solid coupling, Thermal analysis.

\begin{abstract}
This paper elaborates the fluid-structure coupling electromagnetic heat transfer mathematical model of the engine cooling system, combines with finite element method to build the fluid-structure coupled model of water cooling system for the water cooling system, the uniformity of the flow field and temperature field distribution of information, for electromagnetic engine provides the theory basis for structure improvement and cooling performance optimization.
\end{abstract}

\section{Introduction}

Electromagnetic engine is a new kind of engine, it has a simple structure, reliable performance and other advantages, the electromagnetic coil is the core component, coil temperature too high can lead to internal resistance is big, the engine can use efficiency to reduce; The internal heat dissipation of the coil is very poor, and its central position is more easily fused than the outside. The optimization design of the cooling system of electromagnetic coil is the key factor to improve the performance of the engine ${ }^{[1]}$. The heat dissipation of the electromagnetic engine mainly depends on the cold medium heat exchange. The cold medium liquid enters the water cooling system and the surface of the electromagnetic coils for heat exchange, and the heat of the surface of the electromagnetic coil is cooled by the current ${ }^{[2]}$.

The method of fluid-solid coupling has been widely used in the research of motor and engine cooling systems ${ }^{[3]}$. Aiming at the research of the motor cooling system, the simulation analysis of the motor system and the cooling system flow path is more ${ }^{[4]}$. According to the research of engine cooling system, the heat transfer performance of different fin structures in the middle cooler is studied. The water cooling system of electromagnetic engine has not been studied in depth. This article uses the FLUNET fluid calculation software for electromagnetic engine as the research object, combined with finite element method to build the fluid-structure coupled model of water cooling system for the water cooling system, the uniformity of the flow field and temperature field distribution of information.

\section{The fluid-solid coupling heat transfer mathematical model}

Electromagnetic engine cooling fluid flow and heat transfer in the system to follow the mass conservation, momentum conservation, energy conservation law of three solid steady temperature field of the thermal conductivity follows the Laplace's equation, the basic equation is as follows:

$$
\begin{gathered}
\frac{\partial(\rho \varphi)}{\partial t}+\operatorname{div}(\rho u \varphi)=\operatorname{div}(\Gamma \operatorname{grad} \varphi)+s \\
\frac{\partial^{2} T}{\partial x^{2}}+\frac{\partial^{2} T}{\partial y^{2}}+\frac{\partial^{2} T}{\partial z^{2}}=0
\end{gathered}
$$

In the formula:s is the momentum square source phase; $\Gamma$ is generalized diffusion coefficient; $u$ is the velocity vector; $\rho$ is fluid density; $T$ is the temperature of the solid.

The mathematical model equations of fluid-solid coupled heat transfer in electromagnetic engine water cooling system are as follows:

$$
\begin{gathered}
\Delta \mathrm{p}=\frac{1}{2} \rho f U_{A \min }^{2} \\
\mathrm{f}=\left(k_{c}+1+\sigma^{2}\right)-\left(1-\sigma^{2}-k_{e}\right) \frac{v_{e}}{v_{i}}+2\left(\frac{v_{e}}{v_{i}}-1\right)+a R e^{b} \frac{A}{A_{C}} \frac{v_{\mu}}{v_{i}}
\end{gathered}
$$


Where:p is the loss of resistance along the path; $f$ is the pressure loss coefficient; $k_{c}$ and $k_{e}$ are pressure loss factors for inlet and outlet respectively; $\rho$ is air density; $U_{A m i n}^{2}$ for minimum of liquid flow rate; $a$ is the friction factor; $b$ is the friction index; $A$ is the heat dissipation area; $A_{C}$ is the minimum flow surface area; $\sigma$ is the minimum flow rate; $R e$ is the Reynolds number; $v_{i}$ and $v_{e}$ are the velocity of cold fluid inlet and outlet; $v_{\mu}$ is the average velocity of the cold fluid.

\section{Fluid-solid coupling analysis}

Coupled field analysis requires that the analysis software itself has multiple field analysis functions, which can simulate the unit of multiple phenomena simultaneously under the unified interface and database. Fluid-solid coupling analysis is based on fluid mechanics analysis module and static structure analysis module, which is analyzed in a unified platform.

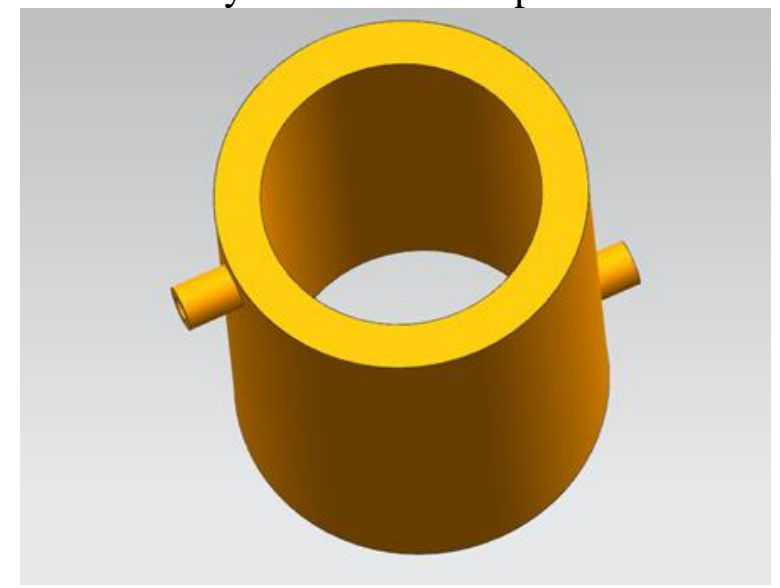

\subsection{Modeling}

Fig.1 water cooling system structure

Fig. 1 shows the schematic diagram of water cooling system. Cooling water after the pump pressure, internal pipe through the inlet into the water cooling system, cooling water heated by electromagnetic electromagnetic coil heat within the engine after the temperature rise sharply, inside the water cooling system and air after heat from the outlet flow back into the water tank. Watercooling system materials is aluminium alloy, aluminium alloy belongs to inverse magnetic materials, magnetic field produced by the action of outside magnetic moment is small, then the heat generated by the magnetic vortex is small, the magnetic permeability of the aluminum alloy is about 1.00022 .

\subsection{Fixed field grid division}

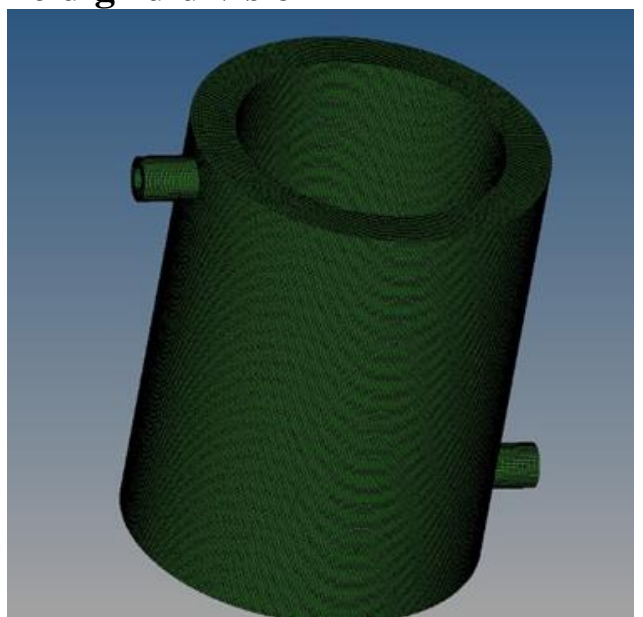

Fig. 2 fixed field grid division

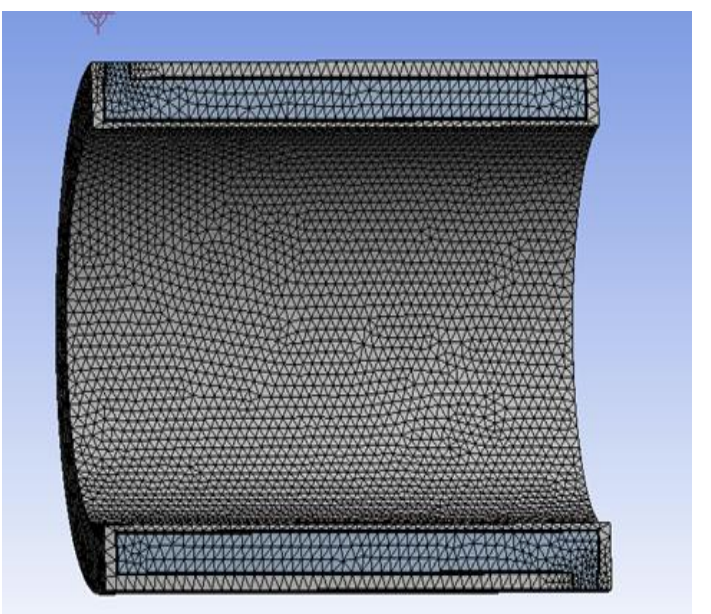

Fig. 3 Flow field grid division

The hypermesh used in this paper is used to divide the grid and optimize the grid with hypermesh. The grid generated by Aotumesh is not ideal. Before the grid division, according to the complex situation of the model, geometric cleaning and geometric classification of the model need to be carried out, and manual optimization is required. Inspect the quality index of grid, through the cleanup tools 
is optimized, as shown in Fig.2, the grid distributed evenly and no grid is unqualified, in combination with grid check data distribution, the ideal grid quality.

\subsection{Flow field grid division}

Flow field in this paper is adopted by the tetrahedron grid meshing, at the same time as the calculating accuracy, grid refinement in the import and export, import and export calculation so that the result is closer to the real value, but should also consider fluid in local grid mesh with Ken can appear too small, could eventually lead to no convergence, even lead to the results of the analysis is not correct, so dividing water cooling system inside the wall, no edges and corners is too small ,as shown in Fig.3.

\subsection{Flow uniformity simulation analysis}

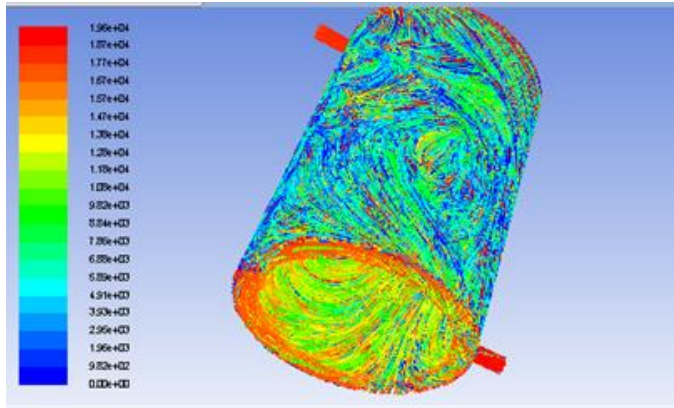

Pathlines Colored by Particle ID

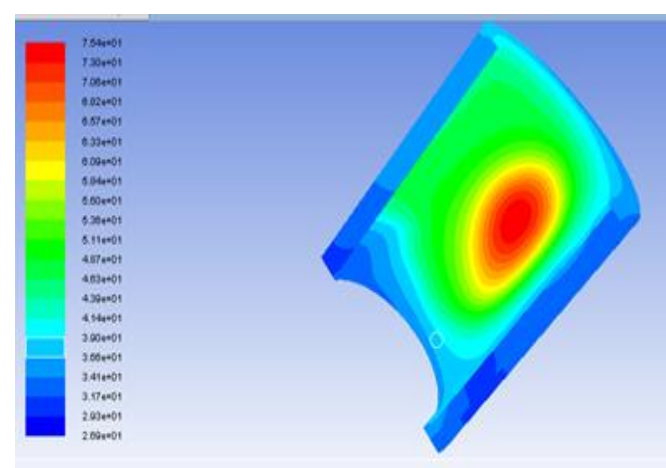

Fig.5 Distribution of temperature field

According to ferri DE man - herm HuoZi equation known, sharply in high-speed fluid karman vortex randomness, water cooling system of flow velocity in import and export of much larger than elsewhere, because the speed is too large, the laminar flow separation. The flow rate of the import and export is about $1.7 \mathrm{~m} / \mathrm{s}$, and there is a swirl flow, as shown in Fig.4.At high speed, the fluid will inevitably have turbulent flow, and then it will turn into a spiral socket. If there is an area of the dead Angle, the area of the dead Angle becomes the dead zone. Dead zone of fluid velocity approximation is 0 , is not conducive to heat transfer, fluid dead area at the same time with high-speed fluid velocity difference is very big, namely according to the theory of laminar flow resistance increase around the dead zone of flow experience, slow.

\subsection{Distribution of temperature field}

As shown in Fig.5, the inner surface temperature of cloud water cooling system, water cooling system inside surface contact with the electromagnetic coil, its temperature is directly decided by the speed of water flow, water is much heat along with fast speed, water velocity region of slow temperature is $73{ }^{\circ} \mathrm{C}$, the location of the high temperature and death.

\section{Summary}

This paper elaborates the fluid-structure coupling electromagnetic heat transfer mathematical model of the engine cooling system, combines with finite element method to build the fluid-structure coupled model of water cooling system for the water cooling system, the uniformity of the flow field and temperature field distribution of information. The results show that the cooling effect of water cooling system is ideal, the internal temperature difference is not large, and there is no serious thermal deformation.

\section{Acknowledgments}

This work is carried out with the support of Scientific Research and Technology Development Program of Guilin (Grant No.2016010201). 


\section{References}

[1] Brown R J S. Connection between formation factor for electrical resistivity and fluid-solid coupling factor in Biot's equations for acoustic waves in fluid-filled porous media[J]. Geophysics, 2012, 45(8):514-514.

[2] Krittian S, Schenkel T, Janoske U, et al. Partitioned fluid-solid coupling for cardiovascular blood flow: validation study of pressure-driven fluid-domain deformation.[J]. Annals of Biomedical Engineering, 2010, 38(8):2676.

[3] Kelly D M, Chen Q, Zang J. PICIN: A Particle-in-Cell Solver for Incompressible Free Surface Flows with Two-Way Fluid-Solid Coupling[J]. Siam Journal on Scientific Computing, 2015, 37(3): B403-B424.

[4] Sanchezricart L, Garcíapeláez J. Fluid-Solid Static Coupling for Saturated Porous Fiber Metals in the Biot's Acoustic Theory[J]. Acta Acustica United with Acustica, 2013, volume 99(99):716728. 